\title{
Genetic variants in RET and risk of Hirschsprung's disease in Southeastern Chinese: a haplotype-based analysis
}

\author{
Jinfa Tou ${ }^{1 *+}$, Li Wang ${ }^{2+}$, Li Liư ${ }^{3}$, Ying Wang ${ }^{3}$, Rong Zhong ${ }^{3}$, Shengyu Duan³, Weiguang Liu', Qixing Xiong ${ }^{1}$,
} Qinglong Gu', Hong Yang ${ }^{5}$, Hui Li ${ }^{2}$

\begin{abstract}
Background: Hirschsprung's disease (HSCR) is a classic oligogenic disorder. Except inactivating mutations of RET, some single nucleotide polymorphisms (SNPS) are identified to be associated with the risk of HSCR. This study was conducted to examine the impact of the haplotypes profile of the reported associated SNPS of RET on the risk of HSCR in a Southeastern Chinese population.

Methods: Genotypes of -5G > A (rs10900296), -1A > C (rs10900297), c135G > A (rs1800858), c1296A > G (rs1800860), and C2307T > G (rs1800861) were analyzed in 123 HSCR patients and 168 controls by polymerase chain reaction amplification and direct sequencing. Associations with risk of HSCR were estimated by odds ratio (OR) and their 95\% confidence intervals (95\% Cl) using logistic regression.

Results: We observed a significantly increased risk of HSCR associated with the RET $-5 A A(O R=17.75,95 \% \mathrm{Cl}=$ 7.34-42.92), -1CC (OR = 10.89, 95\% Cl =3.13-37.85), 135AA (OR = 13.61, 95\% Cl =6.14-30.14), $1296 \mathrm{GG}(\mathrm{OR}=2.40$, $95 \% \mathrm{Cl}=1.38-4.18)$ or $2307 \mathrm{GG}(\mathrm{OR}=9.79,95 \% \mathrm{Cl}=4.28-22.43)$ respectively. The five SNPs were in strong linkage disequilibrium. The haplotype A-C-A-G-G $(O R=5.06,95 \% \mathrm{Cl}=1.97-12.99)$ and diplotype A-C-A-G-G/A-C-A-G-G (OR $=21.08,95 \% \mathrm{Cl}=5.28-84.09)$ was also associated with the increased risk of HSCR, indicating a cumulative effect of these SNPS on the susceptibility of HSCR.
\end{abstract}

Conclusion: These results support the hypothesis that common variations in RET pathway might play an important role in development of HSCR.

\section{Background}

Hirschsprung's disease (HSCR) is a congenital disorder characterized by the absence of enteric ganglion cells in the myenteric (Auerbach's) and submucosal (Meissner's) plexuses of the variable lengths of gastrointestinal tract. The lack of enteric neurons is attributed to the defective proliferation, differentiation, survival, and migration of enteric nervous system (ENS) towards the end of the gut, whereby neural crest cells (NCC) fail to innervate the lower gastrointestinal tract during embryonic development, presenting in clinic with failure to pass meconium, bilious vomiting, enterocolitis-associated

\footnotetext{
*Correspondence: toujingfa@gmail.com

† Contributed equally

'Department of Pediatric Surgery, Children's Hospital Zhejiang University

School of Medicine, Hangzhou, PR China

Full list of author information is available at the end of the article
}

diarrhoea, chronic severe constipation and progressive abdominal distension in the neonatal period [1,2]. Based on the length of the aganglionic segment, it can be classified into three subgroups: short-segment aganglionosis (S-HSCR), long-segment aganglionosis (L-HSCR), and total colonic aganglionsis (TCA). Noteworthy, there is significant racial variation in the incidence of the disease worldwide, which is most prevalent in Asians, including China, about 2.8 per 10,000 live births. The male to female ration (M:F) is around 4:1 among S-HSCR patients and 1:1 among L-HSCR patients. Generally, HSCR most commonly presents sporadically although it can be familial and manifests with low, sex-dependent penetrance and phenotypic variability [2].

HSCR is a heterogenic disorder with a number of genes reported to be associated, most of which involved

\section{C) Biomed Central}


in the signaling pathway implicated in the proper ENS development. RET, a receptor tyrosine kinase, is the major susceptibility gene for HSCR, which locates in 10q11.2 and encodes a transmembrane tyrosine kinase receptor, responsible for triggering a number of downstream signaling transductions [3-5]. There is growing evidence showing that some potentially functional single nucleotide polymorphisms (SNPs) of RET gene could act as low susceptibility factors and modify the phenotype of HSCR, especially in certain combinations of alleles, haplotypes. Specifically, three common SNPs in the coding region of RET, c135G > A in exon2 $(\mathrm{rs} 1800858, \mathrm{~A} 45 \mathrm{~A}), \mathrm{c} 1296 \mathrm{~A}>\mathrm{G}(\mathrm{rs} 1800860, \mathrm{~A} 432 \mathrm{~A})$ in exon7 and c2307T > G (rs1800861, L769L) in exon13, were firstly reported to be associated with HSCR [6-8]. Two promoter polymorphisms $-5 \mathrm{G}>\mathrm{A}$ and $-1 \mathrm{~A}>\mathrm{C}$ (rs10900296 and rs10900297) were also reported to be strongly associated with HSCR and have a significantly lower activity in an in vitro dual-luciferase expression assay conducted by Fitze $G$ and his colleagues [2,9]. Garcia-Barceló, et al [10] further demonstrated that these two SNPs may disrupt a transcriptional factor, TTF1, binding site and then reduce transcription capacity from the promoter. However, to our best knowledge, looking at these 5 particular SNPs all at once in haplotype profile in the Chinese Han population and their effects on the risk of HSCR have not been fully described.

In this study, we conducted a large case-control study to evaluate the contribution of the genotypes and haplotypes of these aforementioned five SNPs in the pathogenesis of sporadic HSCR in a Southeastern Chinese population.

\section{Methods}

\section{Study subjects}

This case-control study consisted of 123 sporadic HSCR (males: 75.6\%) and 194 controls (males: 73.7\%). Cases were histological diagnosed with either biopsy or surgical resection material for absence of enteric plexuses in Children's Hospital Affiliated to Zhejiang University Medical School, among which twelve (10\%) of patients were affected with long-segment aganglionosis (LSA) and the rest with short-segment aganglionosis (SSA). Controls were normal children taking physical examination in the same hospital during the same time period as the patients were enrolled and frequency matched to cases by sex and age. Both cases and controls were Chinese Han in Zhejiang and informed consent was obtained from their parents. The study was approved by the Institutional Review Board of the Children's Hospital Affiliated to Zhejiang University Medical School.

\section{Polymorphism Analysis}

Genomic DNA was extracted from blood sample of all study subjects by using QIAamp-Blood Kit (Qiagen, Hilden, Germany). Genotypes were determined by PCR and direct sequencing without knowledge of the subjects' case/control status. Fifteen percent of the masked sample was randomly selected and genotyped twice by different people independently. The reproducibility was $100 \%$.

RET SNPs $(-5 \mathrm{G}>\mathrm{A},-1 \mathrm{~A}>\mathrm{C}, \mathrm{c} 135 \mathrm{G}>\mathrm{A}, \mathrm{c1296A}>\mathrm{G}$, and $\mathrm{c} 2307 \mathrm{~T}>\mathrm{G}$ ) were genotyped as described previously $[6,11,12]$. PCR primers for RET $-5 \mathrm{G}>\mathrm{A}$ and $-1 \mathrm{~A}>$ C were 5'-CCCGCACTGAGCTCCTAC-3' and 5'-GG ACGTCGCCTTCGCCAT-3', and 5'-TCT CGAGGGATGCTTACTGG-3' and 5'-GGCTCCGGTTAAGGTAGAGG-3' for c135G > A, 5'-CCCTGGTGGGGCATTT GTGC-3' and 5'-GGGTGGTTGGAAACTGATGG-3' for c1296 A > G and 5'-GAACTTGGGCAAGGCGATGC-3' and 5'-ACCCTGCAGCTGGCCTTAC-3' for c2307T > G, respectively. Amplication of the fragments was accomplished under a 25 -ul reaction mixture with $\sim 100 \mathrm{ng}$ template DNA, $0.5 \mathrm{uM}$ each primer, $0.2 \mathrm{mM}$ each dNTP, $2.0 \mathrm{mM} \mathrm{MgCl} 2$ and 1.0 units of Tag DNA polymerase with $1 \times$ reaction buffer (Applied Biosystems, Foster City, CA). The PCR profile consisted of an initial melting step of 5 minutes at $95^{\circ} \mathrm{C}$, followed by 35 cycles of 40 seconds at $95^{\circ} \mathrm{C}, 30$ seconds at $63^{\circ} \mathrm{C}\left(57^{\circ} \mathrm{C}\right.$ for c135G $>\mathrm{A}$ and c2307T > G), and 45 second at $72^{\circ} \mathrm{C}$, and a final elongation step of 7 minutes at $72^{\circ} \mathrm{C}$. PCR products were then sequenced using an ABI PRISM ${ }^{\circledR}$ Big Dye ${ }^{\mathrm{TM}}$ Terminator v3.1 Cycle sequencing kit (Applied Biosystems, Foster City, CA) and an ABI 3130 automated sequencer according to the manufacturer's instructions.

\section{Statistical analysis}

Chi-square test was used to compare the allele and genotype frequencies between cases and controls, as well as LSA and SSA. The associations between the genotype and the risk of HSCR were estimated by odds ratios (ORs) and their 95\% confidence interval (CI), which was calculated by unconditional logistic regression adjusted for gender. PHASE v2.1 [13] was used to estimate the haplotype frequencies composed of five SNP pairs, respectively, and their difference between patients and controls. Diplotype were defined by two haplotypes of each individual. Bonferroni adjustment was used to adjust multiple comparison and $\mathrm{p}$ values were considered significantly at a level of 0.003 , to allow for the testing of 5 partially correlated markers using 3 different inheritance model (dominant, recessive, and additive model). All of the statistical analyses were performed using Statistical Analysis System software (version 9.12; SAS Institute, Cary, NC). 


\section{Results}

Association of RET SNPs with the risk of HSCR

All the five markers in the controls conformed to HWE ( $P>0.05$, data not shown). The frequency of A allele in $-5 \mathrm{G}>\mathrm{A}, \mathrm{C}$ allele in $-1 \mathrm{~A}>\mathrm{C}$, A allele in 135G > A, G allele in 1296A > G and $G$ allele in 2307T > $G$ in cases were $82.5 \%, 88.6 \%, 78.9 \%, 90.7 \%$ and $82.1 \%$, which was higher than that of $45.5 \%, 63.1 \%, 43.8 \%, 80.4 \%$ and $49.7 \%$ in controls, with the $P$ for Cochran-Armitage trend test of $1.12 \times 10^{-16}, 3.43 \times 10^{-11}, 4.20 \times 10^{-15}, 5.70 \times 10^{-4}$, and $2.32 \times 10^{-14}$, respectively. All those markers were shown to be strongly associated with the HSCR risk (Table 1). None of these SNPs were significantly different between LSA and SSA patients (data not shown).

Unconditional logistic regression model was used to estimate RET SNPs effect (additive, dominant, or recessive) according to the smallest AIC (Akaike information criteria) value. Subjects carrying the RET -5AA, -1CC, 135AA or 2307 GG genotype had a 17.75 -fold $(95 \% \mathrm{CI}=$ 7.34-42.92), 10.89-fold (95\% CI = 3.13-37.85), 13.61-fold (95\% CI $=6.14-30.14)$ or 9.79 -fold $(95 \% \mathrm{CI}=4.28$ 22.43) elevated risk for the development of HSCR, compared with the counterpart wild genotype, respectively. The much higher OR value in the present study and previously published association studies indicated that HSCR is a classic oligogenic disorder, which is different from the common complex diseases, such as sporadic cancers, cardiovascular diseases and type 2 diabetes with OR less than 2,. Because the frequencies of wild genotype for $1296 \mathrm{~A}>\mathrm{G}$ were low, we combined it with heterozygous-type genotype together for analysis. Similarly, the homozygous RET 1296GG genotype was also linked to risk of HSCR, with the OR of 2.40 (95\% CI = 1.38 4.18) compared with the AA or AG genotype.

\section{RET haplotypes and risk of HSCR}

We further analyzed the association of the haplotypes (MAF > 0.05) comprising the five HSCR-associated SNPs with HSCR risk. The haplotype and diplotype frequencies are presented in Table 2 and LD analysis showed that the 5 SNPs are part of the same haplotype block in Chinese (D'> 0.6). The haplotype A-C-A-G-G, was highly associated with an increased risk of HSCR $(\mathrm{OR}=5.06,95 \% \mathrm{CI}=1.97-12.99, P=0.001)$. Intriguingly, the haplotype composited with more HSCR-risk allele associated with more elevated risk of HSCR, indicating there is a cumulative effect of these 5 SNPs on the risk of $\operatorname{HSCR}\left(P=7.33 \times 10^{-17}\right.$, for Cochran-Armitage trend test). We also investigated the associations between RET diplotypes and risk of HSCR. Since the low frequencies of haplotype G-A-G-A-T and G-A-G$\mathrm{G}-\mathrm{T}$, we combined them as reference in the following analysis. In consistent with the results from haplotype, the genetic risk of HSCR was increased in subjects with less copies of the G-A-G-A(G)-T haplotype and more copies of the A-C-A-G-G haplotype, with the $P$ values for the Cochran-Armitage test of $3.09 \times 10^{-15}$. Only diplotype with two copy of risk haplotype, A-C-A-G-G/ A-C-A-G-G, was found to be associated with increased risk of HSCR $(\mathrm{OR}=21.08,95 \% \mathrm{CI}=5.28-84.09)$, when compared with wild type of diplotype, suggesting a haplotype-dosage effect in the genetic susceptibility of HSCR.

\section{Discussion}

This genetic epidemiological study investigated whether genetic polymorphisms in RET, alone or in combination, could have effects on the risk of HSCR in a Southeastern Chinese population. In this case-control study including

Table 1 Genotype distribution of the RET gene polymorphisms in case-control

\begin{tabular}{|c|c|c|c|c|}
\hline Polymorphism & Genotypes & Controls $\mathrm{n}=168$ & cases $n=123$ & $\mathrm{OR}^{b}(95 \% \mathrm{Cl}) ; P$ \\
\hline \multirow[t]{3}{*}{$-5 \mathrm{G}>\mathrm{A}, \mathrm{rs} 10900296^{a}$} & GG & $50(29.8)$ & $7(5.7)$ & Reference \\
\hline & GA & $83(49.4)$ & $29(23.9)$ & $2.50(1.02-6.12) ; 0.046$ \\
\hline & $\mathrm{AA}$ & $35(20.8)$ & $87(67.4)$ & $17.75(7.34-42.92)_{i}<0.001$ \\
\hline \multirow[t]{3}{*}{$-1 \mathrm{~A}>\mathrm{C}, \mathrm{rs} 10900297^{b}$} & $\mathrm{AA}$ & $22(13.1)$ & $3(2.4)$ & Reference \\
\hline & $A C$ & $80(47.6)$ & $22(17.9)$ & $2.02(0.55-7.38) ; 0.288$ \\
\hline & CC & $66(39.3)$ & $98(79.7)$ & $10.89(3.13-37.85)_{i}<0.001$ \\
\hline \multirow[t]{3}{*}{$\mathrm{c} 135 \mathrm{G}>\mathrm{A}, \mathrm{rs} 1800858^{c}$} & GG & $52(31.0)$ & $10(8.1)$ & Reference \\
\hline & GA & $85(50.6)$ & $32(26.0)$ & 1.95 (0.89-4.32); 0.095 \\
\hline & $\mathrm{AA}$ & $31(18.4)$ & $81(65.9)$ & $13.61(6.14-30.14)_{i}<0.001$ \\
\hline \multirow[t]{3}{*}{$\mathrm{C} 1296 \mathrm{~A}>\mathrm{G}, \mathrm{rs} 1800860^{d}$} & $\mathrm{AA}$ & $6(3.6)$ & $0(0.0)$ & Reference \\
\hline & $A G$ & $54(32.1)$ & $23(18.7)$ & \\
\hline & GG & $108(64.3)$ & $100(81.3)$ & $2.40(1.38-4.18) ; 0.002$ \\
\hline \multirow[t]{3}{*}{$\mathrm{c} 2307 \mathrm{~T}>\mathrm{G}, \mathrm{rs} 1800861^{e}$} & $\pi$ & $37(22.0)$ & $9(7.3)$ & Reference \\
\hline & $\mathrm{TG}$ & $95(56.6)$ & $26(21.1)$ & $1.08(0.46-2.53) ; 0.853$ \\
\hline & GG & $36(21.4)$ & $88(71.6)$ & $9.79(4.28-22.43):<0.001$ \\
\hline
\end{tabular}

$a_{p}=1.12 \times 10^{-16},{ }^{b} p=3.43 \times 10^{-11},{ }^{c} p=4.20 \times 10^{-15},{ }^{d} p=5.70 \times 10^{-4},{ }^{e} p=2.32 \times 10^{-14}$, for Cochran-Armitage trend test. 
Table 2 Frequencies and counts of RET haplotypes and diplotypes comprising -5G > A, -1A > C, c135G > A, c1296A > G and C2307T > G

\begin{tabular}{|c|c|c|c|c|c|}
\hline Haplotypes $^{a}$ & \multicolumn{2}{|c|}{ Patients (246 chromosomes) } & \multicolumn{2}{|c|}{ Controls (336 chromosomes) } & \multirow[t]{2}{*}{$\mathrm{OR}^{b}(95 \% \mathrm{Cl}) ; P$} \\
\hline$(-5 ;-1 ; c 135 ;$ c1296; c2307) & $\%$ & numbers & $\%$ & numbers & \\
\hline G-A-G-A-T & 2.4 & 6 & 6.0 & 20 & reference $^{b}$ \\
\hline G-A-G-G-T & 8.1 & 20 & 25.3 & 85 & $0.78(0.28-2.20) ; 0.641$ \\
\hline G-C-G-G-T & 1.6 & 4 & 8.3 & 28 & $0.48(0.12-1.91) ; 0.294$ \\
\hline$A-C-A-A-G$ & 3.3 & 8 & 3.6 & 12 & $2.22(0.62-7.97) ; 0.221$ \\
\hline$A-C-A-G-T$ & 3.3 & 8 & 2.7 & 9 & 2.93 (0.78-10.99); 0.111 \\
\hline A-C-A-G-G & 72.0 & 177 & 34.5 & 116 & 5.06 (1.97-12.99); 0.001 \\
\hline Diplotypes & \multicolumn{2}{|c|}{ Patients (123 subjects) } & \multicolumn{2}{|c|}{ Controls (168 subjects) } & \\
\hline G-A-G-A(G)-T/G-A-G-A(G)-T & 2.4 & 3 & 7.7 & 13 & reference $^{c}$ \\
\hline G-A-G-A(G)-T/others ${ }^{d}$ & 4.9 & 6 & 19.6 & 33 & $0.79(0.17-3.64) ; 0.761$ \\
\hline others/others & 3.3 & 4 & 11.9 & 20 & $0.87(0.17-4.54) ; 0.869$ \\
\hline G-A-G-A(G)-T/A-C-A-G-G & 11.4 & 14 & 27.4 & 46 & $1.30(0.32-5.23) ; 0.712$ \\
\hline others/A-C-A-G-G & 23.6 & 29 & 25.0 & 42 & $2.98(0.78-11.42) ; 0.111$ \\
\hline A-C-A-G-G/A-C-A-G-G & 54.4 & 67 & 8.4 & 14 & $21.08(5.28-84.09)_{i}<0.001$ \\
\hline
\end{tabular}

${ }^{a}$ haplotypes with frequencies less than $5 \%$ were not shown.

${ }^{b} p=7.33 \times 10^{-17},{ }^{c} p=3.09 \times 10^{-15}$, for Cochran-Armitage trend test.

${ }^{d}$ represented not G-A-G-A(G)-T or A-C-A-G-G haplotypes.

123 HSCR cases and 168 age- and sex- frequencymatched controls, we demonstrated that five polymorphisms in RET gene, including two SNPs in the regulatory region and three SNPs in the coding region, had substantial impact on risk of HSCR, separately or collectively. Subjects carrying the homozygous variant genotypes of $-5 \mathrm{G}>\mathrm{A},-1 \mathrm{~A}>\mathrm{C}, \mathrm{c} 135 \mathrm{G}>\mathrm{A}, \mathrm{c} 1296 \mathrm{~A}>\mathrm{G}$ or c2307T > G SNPs were at an increased risk for developing HSCR. Moreover, we also observed cumulative effects of these five polymorphisms on HSCR risk, demonstrating the haplotype composited with more HSCR-risk alleles rendered the hosts more susceptible to HSCR. To our best knowledge, this is the first study to investigate the contribution of the haplotypes of these five SNPs to the pathogenesis of sporadic HSCR in Chinese population. It lends supports to the previous findings that these SNPs were associated with the risk of HSCR [1,2,7,10,14-16].

Liu CP et al. reported C135 G/A and C1296 A/G at exon 2 and the combination of AG of these two SNPs were associated with susceptibility of HSCR in Southeast Chinese population $[17,18]$. Also, they observed these two SNPs were in LD with two other coding SNPs at exon 14 and exon 15 in the same population. Our results expanded their findings and found that C135G/A and C1296 A/G were in LD with the two functional SNPs $(-5 G>A$ and $-1 \mathrm{~A}>\mathrm{C}$ ) in the promoter region and $\mathrm{C} 2307 \mathrm{~T}>\mathrm{G}$ in exon 13. Furthermore, we observed $54.4 \%$ of the cases but only 8.4\% of the controls carried A-C-A-G-G/A-C-A-G-G diplotype, with a more than 21 -fold increased risk of HSCR, indicating a cumulative effect of the A-C-A-G-G combination on the susceptibility of HSCR and A-C-A-GG may represent the core of the susceptibility allele.
Intriguingly, we found that the frequencies of risk alleles of-5G > A, -1A > C, c135G > A, c1296A > G or c2307T > G in Chinese Han were much higher than those in European [19], partially explaining the relatively higher incidence of HSCR in Chinese. In conclusion, we observed a strong association between a haplotype, comprising the 3 most common SNPs in coding region and two in promoter region, and HSCR risk. These results support the hypothesis that common variations in RET pathway play an important role in pathogenesis of HSCR and might provide clues to develop screening and surveillance strategies.

\section{Conclusion}

These results support the hypothesis that common variations in RET pathway might play an important role in development of HSCR.

\section{Acknowledgements}

We would like to express our gratitude to all the subjects who participated in the study, especially Xiaoping Miao for scientific editing this manuscript. This work was supported by research grants from Program for New Century Excellent Talents in University NCET-10-0388 and the Fundamental Research Funds for the Central Universities 2010QN005.

\section{Author details}

'Department of Pediatric Surgery, Children's Hospital Zhejiang University School of Medicine, Hangzhou, PR China. ${ }^{2}$ Department of Epidemiology and Biostatistics, Institute of Basic Medical Sciences, Chinese Academy of Medical Sciences and Peking Union Medical College, Beijing, PR China. ${ }^{3}$ Department of Epidemiology and Biostatistics and MOE Key Lab of Environment and Health, School of Public Health, Tongji Medical College, Huazhong University of Science and Technology, Wuhan, PR China. ${ }^{4}$ Department of Otolaryngology, Capital Pediatric Institute, Beijing, PR China. ${ }^{5}$ Department of Gastroenterology, Peking Union Medical College Hospital, Chinese Academy of Medical Sciences, Beijing, PR China. 


\section{Authors' contributions}

JT, LW, LL, YW and RZ designed the study; WL, OX, OG, SD, HY and HL collected blood samples and epidemiological data and performed the experiments; JT and LW performed the statistical analysis and wrote the paper.

\section{Competing interests}

The authors declare that they have no competing interests.

Received: 14 October 2010 Accepted: 25 February 2011

Published: 25 February 2011

\section{References}

1. Tam PK, Garcia-Barcelo M: Genetic basis of Hirschsprung's disease. Pediatr Surg Int 2009, 25(7):543-558.

2. Amiel J, Sproat-Emison E, Garcia-Barcelo M, Lantieri F, Burzynski G, Borrego S, Pelet A, Arnold S, Miao X, Griseri P, et al: Hirschsprung disease, associated syndromes and genetics: a review. J Med Genet 2008, 45(1):1-14.

3. Angrist M, Kauffman E, Slaugenhaupt SA, Matise TC, Puffenberger EG, Washington SS, Lipson A, Cass DT, Reyna T, Weeks DE, et al: A gene for Hirschsprung disease (megacolon) in the pericentromeric region of human chromosome 10. Nat Genet 1993, 4(4):351-356.

4. Iwashita T, Kruger GM, Pardal R, Kiel MJ, Morrison SJ: Hirschsprung disease is linked to defects in neural crest stem cell function. Science 2003, 301(5635):972-976.

5. Natarajan D, Marcos-Gutierrez C, Pachnis V, de Graaff E: Requirement of signalling by receptor tyrosine kinase RET for the directed migration of enteric nervous system progenitor cells during mammalian embryogenesis. Development 2002, 129(22):5151-5160.

6. Borrego S, Ruiz A, Saez ME, Gimm O, Gao X, Lopez-Alonso M, Hernandez A, Wright FA, Antinolo G, Eng C: RET genotypes comprising specific haplotypes of polymorphic variants predispose to isolated Hirschsprung disease. J Med Genet 2000, 37(8):572-578.

7. Fitze G, Cramer J, Ziegler A, Schierz M, Schreiber M, Kuhlisch E, Roesner D, Schackert HK: Association between C135G/A genotype and RET protooncogene germline mutations and phenotype of Hirschsprung's disease. Lancet 2002, 359(9313):1200-1205.

8. Burzynski GM, Nolte IM, Bronda A, Bos KK, Osinga J, Plaza Menacho I, Twigt B, Maas S, Brooks AS, Verheij JB, et al: Identifying candidate Hirschsprung disease-associated RET variants. Am J Hum Genet 2005, 76(5):850-858.

9. Sancandi M, Griseri P, Pesce B, Patrone G, Puppo F, Lerone M, Martucciello G, Romeo G, Ravazzolo R, Devoto M, et al: Single nucleotide polymorphic alleles in the $5^{\prime}$ region of the RET proto-oncogene define a risk haplotype in Hirschsprung's disease. J Med Genet 2003, 40(9):714-718.

10. Garcia-Barcelo M, Ganster RW, Lui VC, Leon TY, So MT, Lau AM, Fu M, Sham MH, Knight J, Zannini MS, et al: TTF-1 and RET promoter SNPs: regulation of RET transcription in Hirschsprung's disease. Hum Mol Genet 2005, 14(2):191-204.

11. Brooks AS, Bertoli-Avella AM, Burzynski GM, Breedveld GJ, Osinga J, Boven LG, Hurst JA, Mancini GM, Lequin MH, de Coo RF, et al: Homozygous nonsense mutations in KIAA1279 are associated with malformations of the central and enteric nervous systems. Am J Hum Genet 2005, 77(1):120-126.

12. Miao X, Garcia-Barcelo MM, So MT, Leon TY, Lau DK, Liu TT, Chan EK, Lan LC, Wong KK, Lui VC, et al: Role of RET and PHOX2B gene polymorphisms in risk of Hirschsprung's disease in Chinese population. Gut 2007, 56(5):736.

13. Stephens M, Donnelly P: A comparison of bayesian methods for haplotype reconstruction from population genotype data. Am J Hum Genet 2003, 73(5):1162-1169.

14. Griseri P, Bachetti T, Puppo F, Lantieri F, Ravazzolo R, Devoto M, Ceccherini I: A common haplotype at the $5^{\prime}$ end of the RET protooncogene, overrepresented in Hirschsprung patients, is associated with reduced gene expression. Hum Mutat 2005, 25(2):189-195

15. Miao X, Leon TY, Ngan ES, So MT, Yuan ZW, Lui VC, Chen Y, Wong KK, Tam PK, Garcia-Barcelo M: Reduced RET expression in gut tissue of individuals carrying risk alleles of Hirschsprung's disease. Hum Mol Genet 2010, 19(8):1461-1467.
16. Fitze G, Schreiber M, Kuhlisch E, Schackert HK, Roesner D: Association of RET protooncogene codon 45 polymorphism with Hirschsprung disease. Am J Hum Genet 1999, 65(5):1469-1473.

17. Liu C, Jin L, Li H, Lou J, Luo C, Zhou X, Li JC: RET polymorphisms and the risk of Hirschsprung's disease in a Chinese population. J Hum Genet 2008 53(9):825-833.

18. Liu CP, Tang QQ, Lou JT, Luo CF, Zhou XW, Li DM, Chen F, Li X, Li JC: Association analysis of the RET proto-oncogene with Hirschsprung disease in the Han Chinese population of southeastern China. Biochem Genet 2010, 48(5-6):496-503.

19. Emison ES, McCallion AS, Kashuk CS, Bush RT, Grice E, Lin S, Portnoy ME, Cutler DJ, Green ED, Chakravarti A: A common sex-dependent mutation in a RET enhancer underlies Hirschsprung disease risk. Nature 2005, 434(7035):857-863.

\section{Pre-publication history}

The pre-publication history for this paper can be accessed here: http://www.biomedcentral.com/1471-2350/12/32/prepub

doi:10.1186/1471-2350-12-32

Cite this article as: Tou et al: Genetic variants in RET and risk of Hirschsprung's disease in Southeastern Chinese: a haplotype-based analysis. BMC Medical Genetics 2011 12:32.

\section{Submit your next manuscript to BioMed Central and take full advantage of:}

- Convenient online submission

- Thorough peer review

- No space constraints or color figure charges

- Immediate publication on acceptance

- Inclusion in PubMed, CAS, Scopus and Google Scholar

- Research which is freely available for redistribution 